\title{
20
}

\section{Solid lipid curcumin-loaded particles for in vivo fluorescent imaging in humans: a proof of concept*}

\author{
(C) M.A. Gogoleva ${ }^{1}$, B.P. Yakimov ${ }^{1}$, S.A. Rodionov ${ }^{2}$, T.N. Tikhonova ${ }^{3}$, Y.I. Gurfinkel ${ }^{4}$, \\ V.V. Fadeev ${ }^{1}$, J. Lademann ${ }^{5}$, M.E. Darvin ${ }^{5}$, E.A. Shirshin ${ }^{1, \pi}$ \\ ${ }^{1}$ Lomonosov Moscow State University, Faculty of Physics, \\ 119991 Moscow, Russia \\ ${ }^{2}$ N.N. Priorov Central Institute for Traumatology and Orthopedics, \\ 127299 Moscow, Russia \\ ${ }^{3}$ International Laser Center, Lomonosov Moscow State University, \\ 119991 Moscow, Russia \\ ${ }^{4}$ Lomonosov Moscow State University Clinic, \\ 119991 Moscow, Russia \\ ${ }^{5}$ Charité - Universitätsmedizin Berlin, corporate member of Freie Universität Berlin, Humboldt-Universität zu Berlin, and Berlin \\ Institute of Health, Department of Dermatology, Venerology and Allergology, Center of Experimental and Applied Cutaneous \\ Physiology (CCP), \\ 10117 Berlin, Germany \\ Te-mail: shirshin@lid.phys.msu.ru
}

Received January 15, 2019

Revised February 20, 2019

Accepted February 26, 2019

The possibility to use oral intake of fluorescent dyes approved on humans for intravital fluorescence in vivo imaging is attractive due to its simplicity and non-invasive character. Here we investigate the potential of solid lipid curcumin particles (SLCP) for imaging of capillary permeability using fluorescence video capillaroscopy. Curcumin fluorescence corresponding to $0.7 \mu \mathrm{g} / \mathrm{ml}$ concentration was observed from blood plasma after oral consumption of $2 \mathrm{~g}$ of SLCP. However, this signal was $\sim 15 \%$ higher than that of blood plasma intrinsic fluorescence, which was itself at least 10-fold lower than the background signal from pericapillary tissue. By comparing the optical properties and concentration to the case of intravenous injection of sodium fluorescein, we analyzed which parameters of the experiment should be optimized for using oral consumption of fluorescing compounds in intravital in vivo capillaroscopy measurements.

DOI: $10.21883 /$ OS.2019.06.47776.56-19

\footnotetext{
* The 22nd Annual Conference Saratov Fall Meeting 2018 (SFM'18): VI International Symposium „Optics and Biophotonics" and XXII International School for Junior Scientists and Students on Optics, Laser Physics \& Biophotonics, September 24-29, 2018, Saratov, Russia. https://www.sgu.ru/structure/fiz/saratov-fall-meeting/previousconferences/saratov-fall-meeting-2018
}

Полная версия статьи опубликована в английской версии журнала. 